\title{
Linesearch algorithms for split equilibrium problems and nonexpansive mappings
}

\author{
Bui Van Dinh ${ }^{1,2}$, Dang Xuan Son ${ }^{3}$, Liguo Jiao² and Do Sang Kim²
}

\section{"Correspondence:}

dskim@pknu.ac.kr

2Department of Applied

Mathematics, Pukyong National

University, Busan, Korea

Full list of author information is

available at the end of the article

\section{Springer}

\begin{abstract}
In this paper, we first propose a weak convergence algorithm, called the linesearch algorithm, for solving a split equilibrium problem and nonexpansive mapping (SEPNM) in real Hilbert spaces, in which the first bifunction is pseudomonotone with respect to its solution set, the second bifunction is monotone, and fixed point mappings are nonexpansive. In this algorithm, we combine the extragradient method incorporated with the Armijo linesearch rule for solving equilibrium problems and the Mann method for finding a fixed point of an nonexpansive mapping. We then combine the proposed algorithm with hybrid cutting technique to get a strong convergence algorithm for SEPNM. Special cases of these algorithms are also given.

MSC: 47H09; 47J25; 65K10; 65K15; 90C99

Keywords: split equilibrium problem; common fixed point problem; nonexpansive mapping; pseudomonotonicity; projection method; linesearch rule; weak and strong convergence
\end{abstract}

\section{Introduction}

Throughout the paper, unless otherwise is stated, we assume that $\mathbb{H}_{1}$ and $\mathbb{H}_{2}$ are real Hilbert spaces endowed with inner products and induced norms denoted by $\langle\cdot, \cdot\rangle$ and $\|\cdot\|$, respectively, whereas $\mathbb{H}$ refers to any of these spaces. We write $x^{k} \rightarrow x$ or $x^{k} \rightarrow x$ iff $x^{k}$ converges strongly or weakly to $x$, respectively, as $k \rightarrow \infty$. Let $C, Q$ be nonempty closed convex subsets in $\mathbb{H}_{1}, \mathbb{H}_{2}$, respectively, and $A: \mathbb{H}_{1} \rightarrow \mathbb{H}_{2}$ be a bounded linear operator. The split feasible problem (SFP) in the sense of Censor and Elfving [1] is to find $x^{*} \in C$ such that $A x^{*} \in Q$. It turns out that SFP provides a unified framework for the study of many significant real-world problems such as in signal processing, medical image reconstruction, intensity-modulated radiation therapy, et cetera; see, for example, [2-5]. To find a solution of SFP in finite-dimensional Hilbert spaces, a basic scheme proposed by Byrne [6], called the CQ-algorithm, is defined as follows:

$$
x^{k+1}=P_{C}\left(x^{k}+\gamma A^{T}\left(P_{Q}-I\right) A x^{k}\right),
$$

where $I$ is the identity mapping, and $P_{C}$ is projection mapping onto $C$. Xu [7] investigated the SFP setting in infinite-dimensional Hilbert spaces. In this case, the CQ-algorithm be-

(c) 2016 Dinh et al. This article is distributed under the terms of the Creative Commons Attribution 4.0 International License (http://creativecommons.org/licenses/by/4.0/), which permits unrestricted use, distribution, and reproduction in any medium, provided you give appropriate credit to the original author(s) and the source, provide a link to the Creative Commons license, and indicate if changes were made. 
comes

$$
x^{k+1}=P_{C}\left(x^{k}+\gamma A^{*}\left(P_{Q}-I\right) A x^{k}\right),
$$

where $A^{*}$ is the adjoint operator of $A$.

The split feasibility problem when $C$ or $Q$ are fixed points of mappings or common fixed points of mappings and solutions of variational inequality problems was considered in some recent research papers; see, for instance, [8-15]. Recently, Moudafi [16] (see also [17-20]) considered the split equilibrium problems (SEP), more precisely:

Let $f: C \times C \rightarrow \mathbb{R}, g: Q \times Q \rightarrow \mathbb{R}$ be equilibrium bifunctions, that is, $f(x, x)=g(u, u)=0$ for all $x \in C$ and $u \in Q$. The split equilibrium problem takes the form

Find $x^{*} \in C$ such that $x^{*} \in \operatorname{Sol}(C, f)$ and $A x^{*} \in \operatorname{Sol}(Q, g)$,

where $\operatorname{Sol}(C, f)$ is the solution set of the following equilibrium problem $(\operatorname{EP}(C, f))$ :

Find $\bar{x} \in C$ such that $f(\bar{x}, y) \geq 0, \forall y \in C$,

and $\operatorname{Sol}(Q, g)$ is the solution set of the equilibrium problem $\operatorname{EP}(Q, g)$. See $[21,22]$ for more detail on equilibrium problems.

For obtaining a solution of SEP, He [23] introduced an iterative method, which generates a sequence $\left\{x^{k}\right\}$ by

$$
\left\{\begin{array}{l}
x^{0} \in C,\left\{r_{k}\right\} \subset(0,+\infty), \quad \mu>0, \\
f\left(y^{k}, y\right)+\frac{1}{r_{k}}\left\langle y-y^{k}, y^{k}-x^{k}\right\rangle \geq 0, \quad \forall y \in C, \\
g\left(u^{k}, v\right)+\frac{1}{r_{k}}\left\langle v-u^{k}, u^{k}-A y^{k}\right\rangle \geq 0, \quad \forall v \in Q, \\
x^{k+1}=P_{C}\left(y^{k}+\mu A^{*}\left(u^{k}-A y^{k}\right)\right), \quad \forall k \geq 0 .
\end{array}\right.
$$

Under certain conditions on bifunctions and parameters, the author shows that $\left\{x^{k}\right\}$, $\left\{y^{k}\right\}$ weakly converges to a solution of SEP, provided that $f$ and $g$ are monotone on $C$ and $Q$, respectively.

On the other hand, many researchers have been proposed numerical algorithms for finding a common element of the set of solutions of monotone equilibrium problems and the set of fixed points of nonexpansive mappings; see, for example, [24-26] and the references therein.

This paper focuses mainly on a split equilibrium problem and nonexpansive mapping involving pseudomonotone and monotone equilibrium bifunctions in real Hilbert spaces. In detail, let $f: C \times C \rightarrow \mathbb{R}$ be a pseudomonotone bifunction with respect to its solution set, $g: Q \times Q \rightarrow \mathbb{R}$ be a monotone bifunction, and $S: C \rightarrow C$ and $T: Q \rightarrow Q$ be nonexpansive mappings. The problem considered in this paper can be stated as follows ( $\operatorname{SEPNM}(C, Q, A, f, g, S, T)$ or SEPNM for short):

Find $x^{*} \in C$ such that $x^{*} \in \operatorname{Sol}(C, f) \cap \operatorname{Fix}(S)$ and $A x^{*} \in \operatorname{Sol}(Q, g) \cap \operatorname{Fix}(T)$,

where $\operatorname{Fix}(S)$ and $\operatorname{Fix}(T)$ are the fixed points of the mappings $S$ and $T$, respectively. 
It should be noticed that, under the monotonicity assumption on $f$ and $g$, the solution sets $\operatorname{Sol}(C, f)$ and $\operatorname{Sol}(C, g)$ of the equilibrium problems $\operatorname{EP}(C, f)$ and $\operatorname{EP}(Q, g)$ are closed convex sets whenever $f$ and $g$ are lower semicontinuous and convex with respect to the second variables. In addition, the nonexpansiveness assumption of $S$ and $T$ also implies that $\operatorname{Fix}(S)$ and $\operatorname{Fix}(T)$ are closed convex sets. Hence, $\operatorname{Sol}(C, f) \cap \operatorname{Fix}(S)$ and $\operatorname{Sol}(Q, g) \cap \operatorname{Fix}(T)$ are closed convex sets. However, the main difficulty is that, even if these sets are convex, they are not given explicitly as in a standard mathematical programming problem, and therefore the projection onto those sets cannot be computed, and consequently, available methods (see, e.g., $[2,27,28]$ and the references therein) cannot be applied for solving SEPNM directly.

In this paper, we first propose a weak convergence algorithm for solving SEPNM by using a combination of the extragradient method with Armijo linesearch type rule for an equilibrium problem [29] (see also [30-32] for more detail on extragradient algorithms) and the Mann method [33] (see also [34, 35]) for a fixed point problem. We then combine this algorithm with hybrid cutting technique [36] (see also [37]) to get a strong convergence algorithm for SEPNM.

The paper is organized as follows. The next section presents some preliminary results. A weak convergence algorithm and its special case are presented in Section 3. In the last section, we combine the method presented in Section 3 with the hybrid projection method for obtaining a strong convergence algorithm for SEPNM.

\section{Preliminaries}

Let $\mathbb{H}$ be a real Hilbert space, and $C$ a nonempty closed convex subset of $\mathbb{H}$. By $P_{C}$ we denote the metric projection operator onto $C$, that is,

$$
P_{C}(x) \in C: \quad\left\|x-P_{C}(x)\right\| \leq\|x-y\|, \quad \forall y \in C .
$$

The following well-known results will be used in the sequel.

Lemma 1 Suppose that $C$ is a nonempty closed convex subset in $\mathbb{H}$. Then $P_{C}$ has the following properties:

(a) $P_{C}(x)$ is singleton and well defined for every $x$;

(b) $z=P_{C}(x)$ if and only if $\langle x-z, y-z\rangle \leq 0, \forall y \in C$;

(c) $\left\|P_{C}(x)-P_{C}(y)\right\|^{2} \leq\left\langle P_{C}(x)-P_{C}(y), x-y\right\rangle, \forall x, y \in \mathbb{H}$;

(d) $\left\|P_{C}(x)-P_{C}(y)\right\|^{2} \leq\|x-y\|^{2}-\left\|x-P_{C}(x)-y+P_{C}(y)\right\|^{2}, \forall x, y \in \mathbb{H}$.

Lemma 2 Let $\mathbb{H}$ be a real Hilbert space. Then, for all $x, y \in \mathbb{H}$ and $\alpha \in[0,1]$, we have

$$
\|\alpha x+(1-\alpha) y\|^{2}=\alpha\|x\|^{2}+(1-\alpha)\|y\|^{2}-\alpha(1-\alpha)\|x-y\|^{2} .
$$

Lemma 3 (Opial's condition) For any sequence $\left\{x^{k}\right\} \subset \mathbb{H}$ with $x^{k} \rightarrow x$, we have the inequality

$$
\liminf _{k \rightarrow+\infty}\left\|x^{k}-x\right\|<\liminf _{k \rightarrow+\infty}\left\|x^{k}-y\right\|
$$

for all $y \in \mathbb{H}$ such that $y \neq x$. 
Definition 1 We say that an operator $T: \mathbb{H} \rightarrow \mathbb{H}$ is demiclosed at 0 if, for any sequence $\left\{x^{k}\right\}$ such that $x^{k} \rightarrow x$ and $T x^{k} \rightarrow 0$ as $k \rightarrow \infty$, we have $T x=0$.

It is well known that, for a nonexpansive operator $T: \mathbb{H} \rightarrow \mathbb{H}$, the operator $I-T$ is demiclosed at 0 ; see [38], Lemma 2.

Now, we assume that the equilibrium bifunctions $g: Q \times Q \rightarrow \mathbb{R}$ and $f: C \times C \rightarrow \mathbb{R}$ satisfy the following assumptions, respectively.

\section{Assumption A}

$\left(\mathrm{A}_{1}\right) g$ is monotone on $Q$, that is, $g(u, v)+g(v, u) \leq 0$ for all $u, v \in Q$;

$\left(\mathrm{A}_{2}\right) g(u, \cdot)$ is convex and lower semicontinuous on $Q$ for each $u \in Q$;

$\left(\mathrm{A}_{3}\right)$ for all $u, v, w \in Q$,

$$
\limsup _{\lambda \downarrow 0} g(\lambda w+(1-\lambda) u, v) \leq g(u, v) .
$$

\section{Assumption B}

$\left(\mathrm{B}_{1}\right) f$ is pseudomonotone on $C$, that is, if $f(x, y) \geq 0$ implies $f(y, x) \leq 0$ for all $x, y \in C$;

$\left(\mathrm{B}_{2}\right) f(x, \cdot)$ is convex and subdifferentiable on $C$ for all $x \in C$;

(B) $f$ is jointly weakly continuous on $C \times C$ in the sense that, if $x, y \in C$ and $\left\{x^{k}\right\},\left\{y^{k}\right\} \subset C$ converge weakly to $x$ and $y$, respectively, then $f\left(x^{k}, y^{k}\right) \rightarrow f(x, y)$ as $k \rightarrow+\infty$.

Let $\varphi$ be an equilibrium bifunction defined on $C \times C$. For $x, y \in C$, we denote by $\partial_{2} \varphi(x, y)$ the subgradient of the convex function $\varphi(x, \cdot)$ at $y$, that is,

$$
\partial_{2} \varphi(x, y):=\{\hat{\xi} \in \mathbb{H}: \varphi(x, z) \geq \varphi(x, y)+\langle\hat{\xi}, z-y\rangle, \forall z \in C\} .
$$

In particular,

$$
\partial_{2} \varphi(x, x)=\{\hat{\xi} \in \mathbb{H}: \varphi(x, z) \geq\langle\hat{\xi}, z-x\rangle, \forall z \in C\} .
$$

Let $\Delta$ be an open convex set containing $C$. The next lemma can be considered as an infinite-dimensional version of Theorem 24.5 in [39].

Lemma 4 ([40], Proposition 4.3) Let $\varphi: \Delta \times \Delta \rightarrow \mathbb{R}$ be an equilibrium bifunction satisfying conditions $\left(\mathrm{A}_{1}\right)$ on $\Delta$ and $\left(\mathrm{A}_{2}\right)$ on C. Let $\bar{x}, \bar{y} \in \Delta$, and let $\left\{x^{k}\right\},\left\{y^{k}\right\}$ be two sequences in $\Delta$ converging weakly to $\bar{x}, \bar{y}$, respectively. Then, for any $\varepsilon>0$, there exist $\eta>0$ and $k_{\varepsilon} \in \mathbb{N}$ such that

$$
\partial_{2} \varphi\left(x^{k}, y^{k}\right) \subset \partial_{2} \varphi(\bar{x}, \bar{y})+\frac{\varepsilon}{\eta} B
$$

for every $k \geq k_{\varepsilon}$, where B denotes the closed unit ball in $\mathbb{H}$.

Lemma 5 Let the equilibrium bifunction $\varphi$ satisfy assumptions $\left(\mathrm{A}_{1}\right)$ on $\Delta$ and $\left(\mathrm{A}_{2}\right)$ on $C$, and $\left\{x^{k}\right\} \subset C, 0<\underline{\rho} \leq \bar{\rho},\left\{\rho_{k}\right\} \subset[\underline{\rho}, \bar{\rho}]$. Consider the sequence $\left\{y^{k}\right\}$ defined as

$$
y^{k}=\arg \min \left\{\varphi\left(x^{k}, y\right)+\frac{1}{2 \rho_{k}}\left\|y-x^{k}\right\|^{2}: y \in C\right\} .
$$

If $\left\{x^{k}\right\}$ is bounded, then $\left\{y^{k}\right\}$ is also bounded. 
Proof First, we show that if $\left\{x^{k}\right\}$ converges weakly to $x^{*}$, then $\left\{y^{k}\right\}$ is bounded. Indeed,

$$
y^{k}=\arg \min \left\{\varphi\left(x^{k}, y\right)+\frac{1}{2 \rho_{k}}\left\|y-x^{k}\right\|^{2}: y \in C\right\}
$$

and

$$
\varphi\left(x^{k}, x^{k}\right)+\frac{1}{2 \rho_{k}}\left\|x^{k}-x^{k}\right\|^{2}=0
$$

Therefore,

$$
\varphi\left(x^{k}, y^{k}\right)+\frac{1}{2 \rho_{k}}\left\|y^{k}-x^{k}\right\|^{2} \leq 0, \quad \forall k .
$$

In addition, for all $\hat{\xi}^{k} \in \partial_{2} \varphi\left(x^{k}, x^{k}\right)$, we have

$$
\varphi\left(x^{k}, y^{k}\right)+\frac{1}{2 \rho_{k}}\left\|y^{k}-x^{k}\right\|^{2} \geq\left\langle\hat{\xi}^{k}, y^{k}-x^{k}\right\rangle+\frac{1}{2 \rho_{k}}\left\|y^{k}-x^{k}\right\|^{2} .
$$

This implies

$$
-\left\|\hat{\xi}^{k}\right\|\left\|y^{k}-x^{k}\right\|+\frac{1}{2 \rho_{k}}\left\|y^{k}-x^{k}\right\|^{2} \leq 0 .
$$

Hence,

$$
\left\|y^{k}-x^{k}\right\| \leq 2 \rho_{k}\left\|\hat{\xi}^{k}\right\|, \quad \forall k
$$

Because $\left\{\rho_{k}\right\}$ is bounded, $\left\{x^{k}\right\}$ converges weakly to $x^{*}$ and $\hat{\xi}^{k} \in \partial_{2} \varphi\left(x^{k}, x^{k}\right)$. By Lemma 4 the sequence $\left\{\hat{\xi}^{k}\right\}$ is bounded; combining this with the boundedness of $\left\{x^{k}\right\}$, we get that $\left\{y^{k}\right\}$ is also bounded.

Now let us prove Lemma 5. Suppose that $\left\{y^{k}\right\}$ is unbounded, that is, there exists a subsequence $\left\{y^{k_{i}}\right\} \subseteq\left\{y^{k}\right\}$ such that $\lim _{i \rightarrow \infty}\left\|y^{k_{i}}\right\|=+\infty$. By the boundedness of $\left\{x^{k}\right\}$ this implies that $\left\{x^{k_{i}}\right\}$ is also bounded, and without loss of generality, we may assume that $\left\{x^{k_{i}}\right\}$ converges weakly to some $x^{*}$. By the same argument as before, we get that $\left\{y^{k_{i}}\right\}$ is bounded, a contradiction. Therefore, $\left\{y^{k}\right\}$ is bounded.

The following lemmas are well known in the theory of monotone equilibrium problems.

Lemma 6 ([21]) Let $g$ satisfy Assumption A. Then, for all $\alpha>0$ and $u \in \mathbb{H}$, there exists $w \in Q$ such that

$$
g(w, v)+\frac{1}{\alpha}\langle v-w, w-u\rangle \geq 0, \quad \forall v \in Q .
$$

Lemma 7 ([41]) Under the assumptions of Lemma 6, the mapping $T_{\alpha}^{g}$ defined on $\mathbb{H}$ as

$$
T_{\alpha}^{g}(u)=\left\{w \in Q: g(w, v)+\frac{1}{\alpha}\langle v-w, w-u\rangle \geq 0, \forall v \in Q\right\}
$$

has following properties: 
(i) $T_{\alpha}^{g}$ is single-valued;

(ii) $T_{\alpha}^{g}$ is firmly nonexpansive, that is, for any $u, v \in \mathbb{H}$,

$$
\left\|T_{\alpha}^{g}(u)-T_{\alpha}^{g}(v)\right\|^{2} \leq\left\langle T_{\alpha}^{g}(u)-T_{\alpha}^{g}(v), u-v\right\rangle
$$

(iii) $\operatorname{Fix}\left(T_{\alpha}^{g}\right)=\operatorname{Sol}(Q, g)$;

(iv) $\operatorname{Sol}(Q, g)$ is closed and convex.

Lemma 8 ([23]) Under the assumptions of Lemma 7, for $\alpha, \beta>0$ and $u, v \in \mathbb{H}$, we have

$$
\left\|T_{\alpha}^{g}(u)-T_{\beta}^{g}(v)\right\| \leq\|v-u\|+\frac{|\beta-\alpha|}{\beta}\left\|T_{\beta}^{g}(v)-v\right\|
$$

\section{A weak convergence algorithm}

\section{Algorithm 1}

Initialization. Pick $x^{0} \in C$ and choose the parameters $\beta, \eta, \theta \in(0,1), 0<\underline{\rho} \leq \bar{\rho}$,

$\left\{\rho_{k}\right\} \subset[\underline{\rho}, \bar{\rho}], 0<\underline{\gamma} \leq \bar{\gamma}<2,\left\{\gamma_{k}\right\} \subset[\underline{\gamma}, \bar{\gamma}], 0<\alpha,\left\{\alpha_{k}\right\} \subset[\alpha,+\infty), \mu \in\left(0, \frac{\overline{1}}{\|A\|}\right)$.

Iteration $k(k=0,1,2, \ldots)$. Having $x^{\bar{k}}$, do the following steps:

Step 1 . Solve the strongly convex program

$$
C P\left(x^{k}\right) \quad \min \left\{f\left(x^{k}, y\right)+\frac{1}{2 \rho_{k}}\left\|y-x^{k}\right\|^{2}: y \in C\right\}
$$

to obtain its unique solution $y^{k}$.

If $y^{k}=x^{k}$, then set $u^{k}=x^{k}$ and go to Step 4. Otherwise, go to Step 2 .

Step 2. (Armijo linesearch rule) Find $m_{k}$ as the smallest positive integer number $m$ such that

$$
\left\{\begin{array}{l}
z^{k, m}=\left(1-\eta^{m}\right) x^{k}+\eta^{m} y^{m} \\
f\left(z^{k, m}, x^{k}\right)-f\left(z^{k, m}, y^{k}\right) \geq \frac{\theta}{2 \rho_{k}}\left\|x^{k}-y^{k}\right\|^{2} .
\end{array}\right.
$$

Set $\eta_{k}=\eta^{m_{k}}, z^{k}=z^{k, m_{k}}$.

Step 3. Select $\xi^{k} \in \partial_{2} f\left(z^{k}, x^{k}\right)$ and compute $\sigma_{k}=\frac{f\left(z^{k}, x^{k}\right)}{\left\|\xi^{k}\right\|^{2}}, u^{k}=P_{C}\left(x^{k}-\gamma_{k} \sigma_{k} \xi^{k}\right)$.

Step 4.

$$
\left\{\begin{array}{l}
v^{k}=(1-\beta) u^{k}+\beta S u^{k} \\
w^{k}=T_{\alpha_{k}}^{g} A v^{k}
\end{array}\right.
$$

Step 5. Take $x^{k+1}=P_{C}\left(v^{k}+\mu A^{*}\left(T w^{k}-A v^{k}\right)\right)$ and go to iteration $k$ with $k$ replaced by $k+1$.

Lemma 9 Suppose that $p \in \operatorname{Sol}(C, f), f(x, \cdot)$ is convex and subdifferentiable on $C$ for all $x \in C$ and that $f$ is pseudomonotone on $C$. Then, we have:
(a) The Armijo linesearch rule (3.1) is well defined;
(b) $f\left(z^{k}, x^{k}\right)>0$;
(c) $0 \notin \partial_{2} f\left(z^{k}, x^{k}\right)$; 
(d)

$$
\left\|u^{k}-p\right\| \leq\left\|x^{k}-p\right\|^{2}-\gamma_{k}\left(2-\gamma_{k}\right)\left(\sigma_{k}\left\|\xi^{k}\right\|\right)^{2}
$$

Proof The proof of Lemma 9 when $\mathbb{H}_{1}$ is a finite-dimensional space can be found, for example, in [29]. When its dimension is infinite, it can be done in the same way. So we omit it.

Theorem 1 Let $C$ and $Q$ be two nonempty closed convex subsets in $\mathbb{H}_{1}$ and $\mathbb{H}_{2}$, respectively. Let $S: C \rightarrow C ; T: Q \rightarrow Q$ be nonexpansive mappings, and let bifunctions $g$ and $f$ satisfy Assumptions $\mathrm{A}$ and $\mathrm{B}$, respectively. Let $A: \mathbb{H}_{1} \rightarrow \mathbb{H}_{2}$ be a bounded linear operator with its adjoint $A^{*}$. If $\Omega=\left\{x^{*} \in \operatorname{Sol}(C, f) \cap \operatorname{Fix}(S): A x^{*} \in \operatorname{Fix}(Q, g) \cap \operatorname{Fix}(T)\right\} \neq \emptyset$, then the sequences $\left\{x^{k}\right\},\left\{u^{k}\right\},\left\{v^{k}\right\}$ converge weakly to an element $p \in \Omega$, and $\left\{w^{k}\right\}$ converges weakly to Ap $\in$ $\operatorname{Sol}(Q, g) \cap \operatorname{Fix}(T)$.

Proof Let $x^{*} \in \Omega$. Then $x^{*} \in \operatorname{Sol}(C, f) \cap \operatorname{Fix}(S)$ and $A x^{*} \in \operatorname{Sol}(Q, g) \cap \operatorname{Fix}(T)$.

From Lemma 9(d) we have

$$
\begin{aligned}
\left\|u^{k}-x^{*}\right\|^{2} & \leq\left\|x^{k}-x^{*}\right\|^{2}-\gamma_{k}\left(2-\gamma_{k}\right)\left(\sigma_{k}\left\|\xi^{k}\right\|\right)^{2} \\
& \leq\left\|x^{k}-x^{*}\right\|^{2} .
\end{aligned}
$$

By Step 4 we get

$$
\begin{aligned}
\left\|v^{k}-x^{*}\right\| & =\left\|(1-\beta) u^{k}+\beta S u^{k}-x^{*}\right\| \\
& =\left\|(1-\beta)\left(u^{k}-x^{*}\right)+\beta\left(S u^{k}-S x^{*}\right)\right\| \\
& \leq(1-\beta)\left\|u^{k}-x^{*}\right\|+\beta\left\|S u^{k}-S x^{*}\right\| \\
& \leq(1-\beta)\left\|u^{k}-x^{*}\right\|+\beta\left\|u^{k}-x^{*}\right\| \\
& =\left\|u^{k}-x^{*}\right\| .
\end{aligned}
$$

Thus,

$$
\left\|v^{k}-x^{*}\right\| \leq\left\|u^{k}-x^{*}\right\| \leq\left\|x^{k}-x^{*}\right\| .
$$

Assertions (iii) and (ii) in Lemma 7 imply that

$$
\begin{aligned}
\left\|T_{\alpha_{k}}^{g} A v^{k}-A x^{*}\right\|^{2} & =\left\|T_{\alpha_{k}}^{g} A v^{k}-T_{\alpha_{k}}^{g} A x^{*}\right\|^{2} \\
& \leq\left\langle T_{\alpha_{k}}^{g} A v^{k}-T_{\alpha_{k}}^{g} A x^{*}, A v^{k}-A x^{*}\right\rangle \\
& =\left\langle T_{\alpha_{k}}^{g} A v^{k}-A x^{*}, A v^{k}-A x^{*}\right\rangle \\
& =\frac{1}{2}\left[\left\|T_{\alpha_{k}}^{g} A v^{k}-A x^{*}\right\|^{2}+\left\|A v^{k}-A x^{*}\right\|^{2}-\left\|T_{\alpha_{k}}^{g} A v^{k}-A v^{k}\right\|^{2}\right] .
\end{aligned}
$$

Hence,

$$
\left\|T_{\alpha_{k}}^{g} A v^{k}-A x^{*}\right\|^{2} \leq\left\|A v^{k}-A x^{*}\right\|^{2}-\left\|T_{\alpha_{k}}^{g} A v^{k}-A v^{k}\right\|^{2} .
$$


Because of the nonexpansiveness of the mapping $T$, we receive from the last inequality that

$$
\begin{aligned}
\left\|T w^{k}-A x^{*}\right\|^{2} & =\left\|T T_{\alpha_{k}}^{g} A v^{k}-T A x^{*}\right\|^{2} \\
& \leq\left\|T_{\alpha_{k}}^{g} A v^{k}-A x^{*}\right\|^{2} \\
& \leq\left\|A v^{k}-A x^{*}\right\|^{2}-\left\|T_{\alpha_{k}}^{g} A v^{k}-A v^{k}\right\|^{2} .
\end{aligned}
$$

Using (3.3), we have

$$
\begin{aligned}
\left\langle A\left(v^{k}-x^{*}\right), T w^{k}-A v^{k}\right\rangle= & \left\langle A\left(v^{k}-x^{*}\right)+T w^{k}-A v^{k}-\left(T w^{k}-A v^{k}\right), T w^{k}-A v^{k}\right\rangle \\
= & \left\langle T w^{k}-A x^{*}, T w^{k}-A v^{k}\right\rangle-\left\|T w^{k}-A v^{k}\right\|^{2} \\
= & \frac{1}{2}\left[\left\|T w^{k}-A x^{*}\right\|^{2}+\left\|T w^{k}-A v^{k}\right\|^{2}-\left\|A v^{k}-A x^{*}\right\|^{2}\right] \\
& -\left\|T w^{k}-A v^{k}\right\|^{2} \\
= & \frac{1}{2}\left[\left(\left\|T w^{k}-A x^{*}\right\|^{2}-\left\|A v^{k}-A x^{*}\right\|^{2}\right)-\left\|T w^{k}-A v^{k}\right\|^{2}\right] \\
\leq & -\frac{1}{2}\left\|T_{\alpha_{k}}^{g} A v^{k}-A v^{k}\right\|^{2}-\frac{1}{2}\left\|T w^{k}-A v^{k}\right\|^{2} .
\end{aligned}
$$

By the definition of $x^{k+1}$ we have

$$
\begin{aligned}
\left\|x^{k+1}-x^{*}\right\|^{2} & =\left\|P_{C}\left(v^{k}+\mu A^{*}\left(T w^{k}-A v^{k}\right)\right)-P_{C}\left(x^{*}\right)\right\|^{2} \\
& \leq\left\|\left(v^{k}-x^{*}\right)+\mu A^{*}\left(T w^{k}-A v^{k}\right)\right\|^{2} \\
& =\left\|v^{k}-x^{*}\right\|^{2}+\left\|\mu A^{*}\left(T w^{k}-A v^{k}\right)\right\|^{2}+2 \mu\left\langle v^{k}-x^{*}, A^{*}\left(T w^{k}-A v^{k}\right)\right\rangle \\
& \leq\left\|v^{k}-x^{*}\right\|^{2}+\mu^{2}\left\|A^{*}\right\|^{2}\left\|T w^{k}-A v^{k}\right\|^{2}+2 \mu\left\langle A\left(v^{k}-x^{*}\right), T w^{k}-A v^{k}\right\rangle .
\end{aligned}
$$

In combination with (3.4) and (3.2), the last inequality becomes

$$
\begin{aligned}
\left\|x^{k+1}-x^{*}\right\|^{2} \leq & \left\|v^{k}-x^{*}\right\|^{2}+\mu^{2}\left\|A^{*}\right\|^{2}\left\|T w^{k}-A v^{k}\right\|^{2} \\
& -\mu\left\|T w^{k}-A v^{k}\right\|^{2}-\mu\left\|T_{\alpha_{k}}^{g} A v^{k}-A v^{k}\right\|^{2} \\
= & \left\|v^{k}-x^{*}\right\|^{2}-\mu\left(1-\mu\|A\|^{2}\right)\left\|T w^{k}-A v^{k}\right\|^{2}-\mu\left\|w^{k}-A v^{k}\right\|^{2} \\
\leq & \left\|x^{k}-x^{*}\right\|^{2}-\mu\left(1-\mu\|A\|^{2}\right)\left\|T w^{k}-A v^{k}\right\|^{2}-\mu\left\|w^{k}-A v^{k}\right\|^{2} .
\end{aligned}
$$

In view of (3.2), (3.5), and $\mu \in\left(0, \frac{1}{\|A\|^{2}}\right)$, we get

$$
\left\|x^{k+1}-x^{*}\right\| \leq\left\|v^{k}-x^{*}\right\| \leq\left\|u^{k}-x^{*}\right\| \leq\left\|x^{k}-x^{*}\right\|
$$

and

$$
\mu\left(1-\mu\|A\|^{2}\right)\left\|T w^{k}-A v^{k}\right\|^{2}+\mu\left\|w^{k}-A v^{k}\right\|^{2} \leq\left\|x^{k}-x^{*}\right\|^{2}-\left\|x^{k+1}-x^{*}\right\|^{2} .
$$


Therefore, $\lim _{k \rightarrow+\infty}\left\|x^{k}-x^{*}\right\|$ does exist, and we get from (3.6) and (3.7) that

$$
\begin{aligned}
& \lim _{k \rightarrow+\infty}\left\|x^{k}-x^{*}\right\|=\lim _{k \rightarrow+\infty}\left\|v^{k}-x^{*}\right\|=\lim _{k \rightarrow+\infty}\left\|u^{k}-x^{*}\right\| \quad \text { and } \\
& \lim _{k \rightarrow+\infty}\left\|T w^{k}-A v^{k}\right\|=\lim _{k \rightarrow+\infty}\left\|w^{k}-A v^{k}\right\|=0 .
\end{aligned}
$$

From (3.8) and the inequality

$$
\left\|T w^{k}-w^{k}\right\| \leq\left\|T w^{k}-A v^{k}\right\|+\left\|w^{k}-A v^{k}\right\|
$$

we get

$$
\lim _{k \rightarrow+\infty}\left\|T w^{k}-w^{k}\right\|=0
$$

Besides, Lemma 9(d) implies

$$
\left\|u^{k}-x^{*}\right\|^{2} \leq\left\|x^{k}-x^{*}\right\|^{2}-\gamma_{k}\left(2-\gamma_{k}\right)\left(\sigma_{k}\left\|\xi^{k}\right\|\right)^{2}
$$

Hence,

$$
\begin{aligned}
\gamma_{k}\left(2-\gamma_{k}\right)\left(\sigma_{k}\left\|\xi^{k}\right\|\right)^{2} & \leq\left\|x^{k}-x^{*}\right\|^{2}-\left\|u^{k}-x^{*}\right\|^{2} \\
& =\left(\left\|x^{k}-x^{*}\right\|-\left\|u^{k}-x^{*}\right\|\right)\left(\left\|x^{k}-x^{*}\right\|+\left\|u^{k}-x^{*}\right\|\right) .
\end{aligned}
$$

In view of (3.8), we get

$$
\lim _{k \rightarrow+\infty} \sigma_{k}\left\|\xi^{k}\right\|=0
$$

In addition, by the definition of $u^{k}, u^{k}=P_{C}\left(x^{k}-\gamma_{k} \sigma_{k} \xi^{k}\right)$. We have

$$
\left\|u^{k}-x^{k}\right\| \leq \gamma_{k} \sigma_{k}\left\|\xi^{k}\right\|
$$

So we get from (3.10) that

$$
\lim _{k \rightarrow+\infty}\left\|u^{k}-x^{k}\right\|=0
$$

Using $v^{k}=(1-\beta) u^{k}+\beta S u^{k}$, Lemma 2 , and the nonexpansiveness of $S$, we have

$$
\begin{aligned}
\left\|v^{k}-x^{*}\right\|^{2} & =\left\|(1-\beta) u^{k}+\beta S u^{k}-x^{*}\right\|^{2} \\
& =\left\|(1-\beta)\left(u^{k}-x^{*}\right)+\beta\left(S u^{k}-x^{*}\right)\right\|^{2} \\
& =(1-\beta)\left\|u^{k}-x^{*}\right\|^{2}+\beta\left\|S u^{k}-x^{*}\right\|^{2}-\beta(1-\beta)\left\|S u^{k}-u^{k}\right\|^{2} \\
& =(1-\beta)\left\|u^{k}-x^{*}\right\|^{2}+\beta\left\|S u^{k}-S x^{*}\right\|^{2}-\beta(1-\beta)\left\|S u^{k}-u^{k}\right\|^{2} \\
& \leq(1-\beta)\left\|u^{k}-x^{*}\right\|^{2}+\beta\left\|u^{k}-x^{*}\right\|^{2}-\beta(1-\beta)\left\|S u^{k}-u^{k}\right\|^{2} \\
& =\left\|u^{k}-x^{*}\right\|^{2}-\beta(1-\beta)\left\|S u^{k}-u^{k}\right\|^{2} .
\end{aligned}
$$


Therefore,

$$
\beta(1-\beta)\left\|S u^{k}-u^{k}\right\|^{2} \leq\left\|u^{k}-x^{*}\right\|^{2}-\left\|v^{k}-x^{*}\right\|^{2} .
$$

Combining the last inequality with (3.8), we obtain that

$$
\lim _{k \rightarrow+\infty}\left\|S u^{k}-u^{k}\right\|=0
$$

In addition,

$$
\begin{aligned}
\left\|v^{k}-x^{k}\right\| & \leq\left\|v^{k}-u^{k}\right\|+\left\|u^{k}-x^{k}\right\| \\
& =\alpha\left\|S u^{k}-u^{k}\right\|+\left\|u^{k}-x^{k}\right\| .
\end{aligned}
$$

Therefore, we get from (3.11) and (3.13) that

$$
\lim _{k \rightarrow+\infty}\left\|v^{k}-x^{k}\right\|=0
$$

Because $\lim _{k \rightarrow+\infty}\left\|x^{k}-x^{*}\right\|$ exists, $\left\{x^{k}\right\}$ is bounded. By Lemma $5,\left\{y^{k}\right\}$ is bounded, and consequently $\left\{z^{k}\right\}$ is bounded. By Lemma $4,\left\{\xi^{k}\right\}$ is bounded. Step 3 and (3.10) yield

$$
\lim _{k \rightarrow \infty} f\left(z^{k}, x^{k}\right)=\lim _{k \rightarrow \infty}\left[\sigma_{k}\left\|\xi^{k}\right\|\right]\left\|\xi^{k}\right\|=0 .
$$

We have

$$
\begin{aligned}
0 & =f\left(z^{k}, z^{k}\right)=f\left(z^{k},\left(1-\eta_{k}\right) x^{k}+\eta_{k} y^{k}\right) \\
& \leq\left(1-\eta_{k}\right) f\left(z^{k}, x^{k}\right)+\eta_{k} f\left(z^{k}, y^{k}\right),
\end{aligned}
$$

so, we get from (3.1) that

$$
\begin{aligned}
f\left(z^{k}, x^{k}\right) & \geq \eta_{k}\left[f\left(z^{k}, x^{k}\right)-f\left(z^{k}, y^{k}\right)\right] \\
& \geq \frac{\theta}{2 \rho_{k}} \eta_{k}\left\|x^{k}-y^{k}\right\|^{2} .
\end{aligned}
$$

Combining this with (3.15), we have

$$
\lim _{k \rightarrow \infty} \eta_{k}\left\|x^{k}-y^{k}\right\|^{2}=0
$$

Suppose that $p$ is a weak accumulation point of $\left\{x^{k}\right\}$, that is, there exists a subsequence $\left\{x^{k_{j}}\right\}$ of $\left\{x^{k}\right\}$ such that $x^{k_{j}}$ converges weakly to $p \in C$ as $j \rightarrow+\infty$. Then, it follows from (3.11) and (3.14) that $u^{k_{j}} \rightarrow p, v^{k_{j}} \rightarrow p$, and $A v^{k_{j}} \rightarrow A p$.

Since $\lim _{k \rightarrow+\infty}\left\|w^{k}-A v^{k}\right\|=0$, we deduce that $w^{k_{j}} \rightarrow A p$. Because $\left\{w^{k}\right\} \subset Q$ and $Q$ is closed and convex, we have that $A p \in Q$.

From (3.16) we get

$$
\lim _{i \rightarrow \infty} \eta_{k_{i}}\left\|x^{k_{i}}-y^{k_{i}}\right\|^{2}=0 .
$$

We now consider two distinct cases. 
Case $1 . \lim \sup _{i \rightarrow \infty} \eta_{k_{i}}>0$.

In this case, there exist $\bar{\eta}>0$ and a subsequence of $\left\{\eta_{k_{i}}\right\}$, denoted again by $\left\{\eta_{k_{i}}\right\}$, such that, for some $i_{0}>0, \eta_{k_{i}}>\bar{\eta}$ for all $i \geq i_{0}$. Using this fact and (3.17), we have

$$
\lim _{i \rightarrow \infty}\left\|x^{k_{i}}-y^{k_{i}}\right\|=0
$$

Recall that $x^{k} \rightarrow p$, together with (3.18), implies that $y^{k_{i}} \rightarrow p$ as $i \rightarrow \infty$.

By the definition of $y^{k_{i}}$,

$$
y^{k_{i}}=\arg \min \left\{f\left(x^{k_{i}}, y\right)+\frac{1}{2 \rho_{k_{i}}}\left\|y-x^{k_{i}}\right\|^{2}: y \in C\right\},
$$

we have

$$
0 \in \partial_{2} f\left(x^{k_{i}}, y^{k_{i}}\right)+\frac{1}{\rho_{k_{i}}}\left(y^{k_{i}}-x^{k_{i}}\right)+N_{C}\left(y^{k_{i}}\right)
$$

so there exists $\hat{\xi}^{k_{i}} \in \partial_{2} f\left(x^{k_{i}}, y^{k_{i}}\right)$ such that

$$
\left\langle\hat{\xi}^{k_{i}}, y-y^{k_{i}}\right\rangle+\frac{1}{\rho_{k_{i}}}\left\langle y^{k_{i}}-x^{k_{i}}, y-y^{k_{i}}\right\rangle \geq 0, \quad \forall y \in C .
$$

Combining this with

$$
f\left(x^{k_{i}}, y\right)-f\left(x^{k_{i}}, y^{k_{i}}\right) \geq\left\langle\hat{\xi}^{k_{i}}, y-y^{k_{i}}\right\rangle, \quad \forall y \in C
$$

yields

$$
f\left(x^{k_{i}}, y\right)-f\left(x^{k_{i}}, y^{k_{i}}\right)+\frac{1}{\rho_{k_{i}}}\left\langle y^{k_{i}}-x^{k_{i}}, y-y^{k_{i}}\right\rangle \geq 0, \quad \forall y \in C .
$$

Since

$$
\left\langle y^{k_{i}}-x^{k_{i}}, y-y^{k_{i}}\right\rangle \leq\left\|y^{k_{i}}-x^{k_{i}}\right\|\left\|y-y^{k_{i}}\right\|
$$

from (3.19) we get that

$$
f\left(x^{k_{i}}, y\right)-f\left(x^{k_{i}}, y^{k_{i}}\right)+\frac{1}{\rho_{k_{i}}}\left\|y^{k_{i}}-x^{k_{i}}\right\|\left\|y-y^{k_{i}}\right\| \geq 0 .
$$

Letting $i \rightarrow \infty$, by the weak continuity of $f$ and (3.18), from (3.20) we obtain in the limit that

$$
f(p, y)-f(p, p) \geq 0
$$

Hence,

$$
f(p, y) \geq 0, \quad \forall y \in C
$$

which means that $p$ is a solution of $\operatorname{EP}(C, f)$. 
Case 2. $\lim _{i \rightarrow \infty} \eta_{k_{i}}=0$.

From the boundedness of $\left\{y^{k_{i}}\right\}$, without loss of generality, we may assume that $y^{k_{i}} \rightarrow \bar{y}$ as $i \rightarrow \infty$.

Replacing $y$ by $x^{k_{i}}$ in (3.19), we get

$$
f\left(x^{k_{i}}, y^{k_{i}}\right) \leq-\frac{1}{\rho_{k_{i}}}\left\|y^{k_{i}}-x^{k_{i}}\right\|^{2}
$$

On the other hand, by the Armijo linesearch rule (3.1), for $m_{k_{i}}-1$, we have

$$
f\left(z^{k_{i}, m_{k_{i}}-1}, x^{k_{i}}\right)-f\left(z^{k_{i}, m_{k_{i}}-1}, y^{k_{i}}\right)<\frac{\theta}{2 \rho_{k_{i}}}\left\|y^{k_{i}}-x^{k_{i}}\right\|^{2} .
$$

Combining this with (3.21), we get

$$
f\left(x^{k_{i}}, y^{k_{i}}\right) \leq-\frac{1}{\rho_{k_{i}}}\left\|y^{k_{i}}-x^{k_{i}}\right\|^{2} \leq \frac{2}{\theta}\left[f\left(z^{k_{i}, m_{k_{i}}-1}, y^{k_{i}}\right)-f\left(z^{k_{i}, m_{k_{i}}-1}, x^{k_{i}}\right)\right] .
$$

According to the algorithm, we have $z^{k_{i}, m_{k_{i}}-1}=\left(1-\eta^{m_{k_{i}}-1}\right) x^{k_{i}}+\eta^{m_{k_{i}}-1} y^{k_{i}}$. Since $\eta^{k_{i}, m_{k_{i}}-1} \rightarrow 0$, $x^{k_{i}}$ converges weakly to $p$, and $y^{k_{i}}$ converges weakly to $\bar{y}$, this implies that $z^{k_{i}, m_{k_{i}}-1} \rightarrow p$ as $i \rightarrow \infty$. Beside that, $\left\{\frac{1}{\rho_{k_{i}}}\left\|y^{k_{i}}-x^{k_{i}}\right\|^{2}\right\}$ is bounded, so without loss of generality we may assume that $\lim _{i \rightarrow+\infty} \frac{1}{\rho_{k_{i}}}\left\|y^{k_{i}}-x^{k_{i}}\right\|^{2}$ exists. Hence, in the limit, from (3.22) we get that

$$
f(p, \bar{y}) \leq-\lim _{i \rightarrow+\infty} \frac{1}{\rho_{k_{i}}}\left\|y^{k_{i}}-x^{k_{i}}\right\|^{2} \leq \frac{2}{\theta} f(p, \bar{y})
$$

Therefore, $f(p, \bar{y})=0$ and $\lim _{i \rightarrow+\infty}\left\|y^{k_{i}}-x^{k_{i}}\right\|^{2}=0$. By Case 1 we get $p \in \operatorname{Sol}(C, f)$.

Besides that, (3.13) implies that $\left\|S u^{k_{j}}-u^{k_{j}}\right\| \rightarrow 0$ as $j \rightarrow \infty$; together with $u^{k_{j}} \rightarrow p$ and the demiclosedness of $I-S$, we get $p \in \operatorname{Fix}(S)$.

Therefore,

$$
p \in \operatorname{Sol}(C, f) \cap \operatorname{Fix}(S) \text {. }
$$

Next, we need to show that $A p \in \operatorname{Sol}(Q, g) \cap \operatorname{Fix}(T)$.

Indeed, we have $\operatorname{Sol}(Q, g)=\operatorname{Fix}\left(T_{\beta}^{g}\right)$. So, if $T_{\beta}^{g} A p \neq A p$, then, using Opial's condition, we have

$$
\begin{aligned}
\liminf _{j \rightarrow+\infty}\left\|A v^{k_{j}}-A p\right\| & <\liminf _{j \rightarrow+\infty}\left\|A v^{k_{j}}-T_{\beta}^{g} A p\right\| \\
& =\liminf _{j \rightarrow+\infty}\left\|A v^{k_{j}}-w^{k_{j}}+w^{k_{j}}-T_{\beta}^{g} A p\right\| \\
& \leq \liminf _{j \rightarrow+\infty}\left(\left\|A v^{k_{j}}-w^{k_{j}}\right\|+\left\|T_{\beta}^{g} A p-w^{k_{j}}\right\|\right) .
\end{aligned}
$$

So it follows from (3.8) and Lemma 8 that

$$
\begin{aligned}
\liminf _{j \rightarrow+\infty}\left\|A v^{k_{j}}-A p\right\| & <\liminf _{j \rightarrow+\infty}\left\|T_{\beta}^{g} A p-w^{k_{j}}\right\| \\
& =\liminf _{j \rightarrow+\infty}\left\|T_{\beta}^{g} A p-T_{\alpha_{k_{j}}}^{g} A v^{k_{j}}\right\|
\end{aligned}
$$




$$
\begin{aligned}
& \leq \liminf _{j \rightarrow+\infty}\left\{\left\|A v^{k_{j}}-A p\right\|+\frac{\left|\alpha_{k_{j}}-\beta\right|}{\alpha_{k_{j}}}\left\|T_{\alpha_{k_{j}}}^{g} A v^{k_{j}}-A v^{k_{j}}\right\|\right\} \\
& =\liminf _{j \rightarrow+\infty}\left\{\left\|A v^{k_{j}}-A p\right\|+\frac{\left|\alpha_{k_{j}}-\beta\right|}{\alpha_{k_{j}}}\left\|w^{k_{j}}-A v^{k_{j}}\right\|\right\} \\
& =\liminf _{j \rightarrow+\infty}\left\|A v^{k_{j}}-A p\right\|,
\end{aligned}
$$

a contradiction. Thus, $A p \in \operatorname{Fix}\left(T_{\alpha}^{g}\right)=\operatorname{Sol}(Q, g)$.

Moreover, (3.9) shows that $\lim _{j \rightarrow \infty}\left\|T w^{k_{j}}-w^{k_{j}}\right\|=0$. Combining this with $w^{k_{j}} \rightarrow A p$ and the fact that $I-T$ is demiclosed at 0 , it is immediate that $A p \in \operatorname{Fix}(T)$. Therefore,

$$
A p \in \operatorname{Sol}(Q, g) \cap \operatorname{Fix}(T) .
$$

From (3.23) and (3.24) we obtain that $p \in \Omega$.

To complete the proof, we must show that the whole sequence $\left\{x^{k}\right\}$ converges weakly to $p$. Indeed, if there exists a subsequence $\left\{x^{l_{i}}\right\}$ of $\left\{x^{k}\right\}$ such that $x^{l_{i}} \rightarrow q$ with $q \neq p$, then we have $q \in \Omega$. By Opial's condition this yields

$$
\begin{aligned}
\liminf _{i \rightarrow+\infty}\left\|x^{l_{i}}-q\right\| & <\liminf _{i \rightarrow+\infty}\left\|x^{l_{i}}-p\right\| \\
& =\liminf _{j \rightarrow+\infty}\left\|x^{k}-p\right\| \\
& =\liminf _{j \rightarrow+\infty}\left\|x^{k_{j}}-p\right\| \\
& <\liminf _{j \rightarrow+\infty}\left\|x^{k_{j}}-q\right\| \\
& =\liminf _{i \rightarrow+\infty}\left\|x^{l_{i}}-q\right\|,
\end{aligned}
$$

a contradiction. Hence, $\left\{x^{k}\right\}$ converges weakly to $p$.

Combining this with (3.8), it is immediate that $\left\{u^{k}\right\},\left\{v^{k}\right\}$ also converge weakly to $p$ and $w^{k} \rightarrow A p \in \operatorname{Sol}(Q, g) \cap \operatorname{Fix}(T)$.

A particular case of the problem SEPNM is the split equilibrium problem SEP, that is, $S=I_{\mathbb{H}_{1}}$ and $T=I_{\mathbb{H}_{2}}$. In this case, we have the following linesearch algorithm for SEP.

\section{Algorithm 2}

Initialization. Pick $x^{0} \in C$ and choose the parameters $\eta, \theta \in(0,1), 0<\rho \leq \bar{\rho}$, $\left\{\rho_{k}\right\} \subset[\underline{\rho}, \bar{\rho}], 0<\underline{\gamma} \leq \bar{\gamma}<2,\left\{\gamma_{k}\right\} \subset[\underline{\gamma}, \bar{\gamma}], 0<\alpha,\left\{\alpha_{k}\right\} \subset[\alpha,+\infty), \mu \in\left(0, \frac{1}{\|A\|}\right)$.

Iteration $k(k=0,1,2, \ldots)$. Having $x^{\bar{k}}$, do the following steps:

Step 1 . Solve the strongly convex program

$$
C P\left(x^{k}\right) \quad \min \left\{f\left(x^{k}, y\right)+\frac{1}{2 \rho_{k}}\left\|y-x^{k}\right\|^{2}: y \in C\right\}
$$

to obtain its unique solution $y^{k}$.

If $y^{k}=x^{k}$, then set $u^{k}=x^{k}$ and go to Step 4. Otherwise, go to Step 2 . 
Step 2. (Armijo linesearch rule) Find $m_{k}$ as the smallest positive integer number $m$ such that

$$
\left\{\begin{array}{l}
z^{k, m}=\left(1-\eta^{m}\right) x^{k}+\eta^{m} y^{m}, \\
f\left(z^{k, m}, x^{k}\right)-f\left(z^{k, m}, y^{k}\right) \geq \frac{\theta}{2 \rho_{k}}\left\|x^{k}-y^{k}\right\|^{2} .
\end{array}\right.
$$

Set $\eta_{k}=\eta^{m_{k}}, z^{k}=z^{k, m_{k}}$.

Step 3. Select $\xi^{k} \in \partial_{2} f\left(z^{k}, x^{k}\right)$ and compute $\sigma_{k}=\frac{f\left(z^{k}, x^{k}\right)}{\left\|\xi^{k}\right\|^{2}}, u^{k}=P_{C}\left(x^{k}-\gamma_{k} \sigma_{k} \xi^{k}\right)$.

Step 4. $w^{k}=T_{\alpha_{k}}^{g} A u^{k}$.

Step 5. Take $x^{k+1}=P_{C}\left(u^{k}+\mu A^{*}\left(w^{k}-A u^{k}\right)\right)$ and go to iteration $k$ with $k$ is replaced by $k+1$.

The following corollary is an immediate consequence of Theorem 1.

Corollary 1 Suppose that $g, f$ are bifunctions satisfying Assumptions A and B, respectively. Let $A: \mathbb{H}_{1} \rightarrow \mathbb{H}_{2}$ be a bounded linear operator with its adjoint $A^{*}$. If $\Omega=\left\{x^{*} \in \operatorname{Sol}(C, f)\right.$ : $\left.A x^{*} \in \operatorname{Sol}(Q, g)\right\} \neq \emptyset$, then the sequences $\left\{x^{k}\right\}$ and $\left\{u^{k}\right\}$ converge weakly to an element $p \in \Omega$, and $\left\{w^{k}\right\}$ converges weakly to $A p \in \operatorname{Sol}(Q, g)$.

\section{A strong convergence algorithm}

\section{Algorithm 3}

Initialization. Pick $x^{g} \in C_{0}=C$ and choose the parameters $\beta, \eta, \theta \in(0,1), 0<\underline{\rho} \leq \bar{\rho}$, $\left\{\rho_{k}\right\} \subset[\underline{\rho}, \bar{\rho}], 0<\underline{\gamma} \leq \bar{\gamma}<2,\left\{\gamma_{k}\right\} \subset[\underline{\gamma}, \bar{\gamma}], 0<\alpha,\left\{\alpha_{k}\right\} \subset[\alpha,+\infty), \mu \in\left(0, \frac{1}{\|A\|}\right)$.

Iteration $k(k=0,1,2, \ldots)$. Having $x^{\bar{k}}$, do the following steps:

Step 1 . Solve the strongly convex program

$$
C P\left(x^{k}\right) \quad \min \left\{f\left(x^{k}, y\right)+\frac{1}{2 \rho_{k}}\left\|y-x^{k}\right\|^{2}: y \in C\right\}
$$

to obtain its unique solution $y^{k}$.

If $y^{k}=x^{k}$, then set $u^{k}=x^{k}$ and go to Step 4. Otherwise, go to Step 2 .

Step 2. (Armijo linesearch rule) Find $m_{k}$ as the smallest positive integer number $m$ such that

$$
\left\{\begin{array}{l}
z^{k, m}=\left(1-\eta^{m}\right) x^{k}+\eta^{m} y^{m} \\
f\left(z^{k, m}, x^{k}\right)-f\left(z^{k, m}, y^{k}\right) \geq \frac{\theta}{2 \rho_{k}}\left\|x^{k}-y^{k}\right\|^{2} .
\end{array}\right.
$$

Set $\eta_{k}=\eta^{m_{k}}, z^{k}=z^{k, m_{k}}$.

Step 3. Select $\xi^{k} \in \partial_{2} f\left(z^{k}, x^{k}\right)$ and compute $\sigma_{k}=\frac{f\left(z^{k}, x^{k}\right)}{\left\|\xi^{k}\right\|^{2}}, u^{k}=P_{C}\left(x^{k}-\gamma_{k} \sigma_{k} \xi^{k}\right)$.

Step 4.

$$
\left\{\begin{array}{l}
v^{k}=(1-\beta) u^{k}+\beta S u^{k} \\
w^{k}=T_{\beta_{k}}^{g} A v^{k}
\end{array}\right.
$$

Step 5. $t^{k}=P_{C}\left(v^{k}+\mu A^{*}\left(T w^{k}-A v^{k}\right)\right)$. 
Step 6. Define $C_{k+1}=\left\{x \in C_{k}:\left\|x-t^{k}\right\| \leq\left\|x-v^{k}\right\| \leq\left\|x-x^{k}\right\|\right\}$. Compute $x^{k+1}=P_{C_{k+1}}\left(x^{g}\right)$ and go to iteration $k$ with $k$ is replaced by $k+1$.

Theorem 2 Let $C$ and $Q$ be two nonempty closed convex subsets in $\mathbb{H}_{1}$ and $\mathbb{H}_{2}$, respectively. Let $S: C \rightarrow C ; T: Q \rightarrow Q$ be nonexpansive mappings, and let bifunctions $g$ and $f$ satisfy Assumptions $\mathrm{A}$ and $\mathrm{B}$, respectively. Let $A: \mathbb{H}_{1} \rightarrow \mathbb{H}_{2}$ be a bounded linear operator with its adjoint $A^{*}$. If $\Omega=\left\{x^{*} \in \operatorname{Sol}(C, f) \cap \operatorname{Fix}(S): A x^{*} \in \operatorname{Sol}(Q, g) \cap \operatorname{Fix}(T)\right\} \neq \emptyset$, then the sequences $\left\{x^{k}\right\},\left\{u^{k}\right\},\left\{v^{k}\right\}$ converge strongly to an element $p \in \Omega$, and $\left\{w^{k}\right\}$ converges strongly to Ap $\in$ $\operatorname{Sol}(Q, g) \cap \operatorname{Fix}(T)$.

Proof First, we observe that the linesearch rule (4.1) is well defined by Lemma 9. Let $x^{*} \in \Omega$. From (3.5), (3.12), and (3.2) we have

$$
\begin{aligned}
\left\|t^{k}-x^{*}\right\|^{2} \leq & \left\|v^{k}-x^{*}\right\|^{2}-\mu\left(1-\mu\|A\|^{2}\right)\left\|T w^{k}-A v^{k}\right\|^{2}-\mu\left\|w^{k}-A v^{k}\right\|^{2} \\
\leq & \left\|u^{k}-x^{*}\right\|^{2}-\beta(1-\beta)\left\|S u^{k}-u^{k}\right\|^{2} \\
& -\mu\left(1-\mu\|A\|^{2}\right)\left\|T w^{k}-A v^{k}\right\|^{2}-\mu\left\|w^{k}-A v^{k}\right\|^{2} \\
\leq & \left\|x^{k}-x^{*}\right\|^{2}-\beta(1-\beta)\left\|S u^{k}-u^{k}\right\|^{2} \\
& -\mu\left(1-\mu\|A\|^{2}\right)\left\|T w^{k}-A v^{k}\right\|^{2}-\mu\left\|w^{k}-A v^{k}\right\|^{2} .
\end{aligned}
$$

Since $\mu \in\left(0, \frac{1}{\|A\|^{2}}\right),(4.2)$ implies that

$$
\left\|t^{k}-x^{*}\right\| \leq\left\|v^{k}-x^{*}\right\| \leq\left\|u^{k}-x^{*}\right\| \leq\left\|x^{k}-x^{*}\right\|, \quad \forall k .
$$

Since $x^{*} \in C_{0}$, from(4.3) we get by induction that $x^{*} \in C_{k}$ for all $k \in \mathbb{N}^{*}$ and, consequently, $\Omega \subset C_{k}$ for all $k$.

By setting

$$
D_{k}=\left\{x \in \mathbb{H}_{1}:\left\|x-t^{k}\right\| \leq\left\|x-v^{k}\right\| \leq\left\|x-x^{k}\right\|\right\}, \quad k \in \mathbb{N},
$$

it is clear that $D_{k}$ is closed and convex for all $k$. In addition, $C_{0}=C$ is also closed and convex, and $C_{k+1}=C_{k} \cap D_{k}$. Hence, $C_{k}$ is closed and convex for all $k$.

From the definition of $x^{k+1}$ we have $x^{k+1} \in C_{k+1} \subset C_{k}$ and $x^{k}=P_{C_{k}}\left(x^{g}\right)$, so

$$
\left\|x^{k}-x^{g}\right\| \leq\left\|x^{k+1}-x^{g}\right\| \quad \text { for all } k \text {. }
$$

Since $x^{*} \in C_{k+1}$, this implies that

$$
\left\|x^{k+1}-x^{g}\right\| \leq\left\|x^{*}-x^{g}\right\|
$$

Thus,

$$
\left\|x^{k}-x^{g}\right\| \leq\left\|x^{k+1}-x^{g}\right\| \leq\left\|x^{*}-x^{g}\right\|, \quad \forall k .
$$

Consequently, $\left\{\left\|x^{k}-x^{g}\right\|\right\}$ is nondecreasing and bounded, so $\lim _{k \rightarrow+\infty}\left\|x^{k}-x^{g}\right\|$ does exist. Combining this with (4.3), we obtain that $\left\{t^{k}\right\}$ and $\left\{v^{k}\right\}$ are also bounded. 
For all $m>n$, we have that $x^{m} \in C_{m} \subset C_{n}$ and $x^{n}=P_{C_{n}}\left(x^{g}\right)$. Combining this fact with Lemma 1, we get

$$
\begin{aligned}
\left\|x^{m}-x^{n}\right\|^{2} & \leq\left\|x^{m}-x^{g}\right\|^{2}-\left\|x^{n}-x^{g}\right\|^{2} \\
& =\left(\left\|x^{m}-x^{g}\right\|-\left\|x^{n}-x^{g}\right\|\right)\left(\left\|x^{m}-x^{g}\right\|+\left\|x^{n}-x^{g}\right\|\right) .
\end{aligned}
$$

Since $\lim _{k \rightarrow+\infty}\left\|x^{k}-x^{g}\right\|$ exists, this implies that $\lim _{m, n \rightarrow \infty}\left\|x^{m}-x^{n}\right\|=0$, i.e., $\left\{x^{k}\right\}$ is a Cauchy sequence, so

$$
\lim _{k \rightarrow \infty} x^{k}=p
$$

By Step 6 we get

$$
\left\|t^{k}-x^{k+1}\right\| \leq\left\|v^{k}-x^{k+1}\right\| \leq\left\|x^{k}-x^{k+1}\right\| .
$$

Therefore,

$$
\begin{aligned}
\left\|t^{k}-x^{k}\right\| & \leq\left\|t^{k}-x^{k+1}\right\|+\left\|x^{k+1}-x^{k}\right\| \\
& \leq\left\|x^{k}-x^{k+1}\right\|+\left\|x^{k}-x^{k+1}\right\| \\
& =2\left\|x^{k}-x^{k+1}\right\|
\end{aligned}
$$

and

$$
\begin{aligned}
\left\|v^{k}-x^{k}\right\| & \leq\left\|v^{k}-x^{k+1}\right\|+\left\|x^{k+1}-x^{k}\right\| \\
& \leq\left\|x^{k}-x^{k+1}\right\|+\left\|x^{k}-x^{k+1}\right\| \\
& =2\left\|x^{k}-x^{k+1}\right\| .
\end{aligned}
$$

So, from (4.5), (4.6), and (4.4) we get that

$$
\lim _{k \rightarrow \infty}\left\|t^{k}-x^{k}\right\|=\lim _{k \rightarrow \infty}\left\|v^{k}-x^{k}\right\|=0
$$

In view of (4.2) and (4.7), we have

$$
\begin{aligned}
& \beta(1-\beta)\left\|S u^{k}-u^{k}\right\|^{2}+\mu\left(1-\mu\|A\|^{2}\right)\left\|T w^{k}-A v^{k}\right\|^{2}+\mu\left\|w^{k}-A v^{k}\right\|^{2} \\
& \quad \leq\left\|x^{k}-x^{*}\right\|^{2}-\left\|t^{k}-x^{*}\right\|^{2} \\
& \quad=\left(\left\|x^{k}-x^{*}\right\|+\left\|t^{k}-x^{*}\right\|\right)\left(\left\|x^{k}-x^{*}\right\|-\left\|t^{k}-x^{*}\right\|\right) \\
& \quad \leq\left\|x^{k}-t^{k}\right\|\left(\left\|x^{k}-x^{*}\right\|+\left\|t^{k}-x^{*}\right\|\right) \rightarrow 0 \quad \text { as } k \rightarrow \infty .
\end{aligned}
$$

Since $\beta \in(0,1)$ and $\mu \in\left(0, \frac{1}{\|A\|}\right)$, we deduce from (4.8) that

$$
\begin{aligned}
& \lim _{k \rightarrow+\infty}\left\|S u^{k}-u^{k}\right\|=0, \quad \lim _{k \rightarrow+\infty}\left\|T w^{k}-A v^{k}\right\|=0, \quad \text { and } \\
& \lim _{k \rightarrow+\infty}\left\|w^{k}-A v^{k}\right\|=0 .
\end{aligned}
$$


In addition, from the inequality

$$
\left\|T w^{k}-w^{k}\right\| \leq\left\|T w^{k}-A v^{k}\right\|+\left\|w^{k}-A v^{k}\right\|,
$$

combined with (4.9), we get

$$
\lim _{k \rightarrow+\infty}\left\|T w^{k}-w^{k}\right\|=0
$$

Besides, (3.11), (4.6), and $\lim _{k \rightarrow+\infty} x^{k}=p$ it imply

$$
\lim _{k \rightarrow+\infty} u^{k}=p, \quad \lim _{k \rightarrow+\infty} v^{k}=p
$$

Since

$$
\begin{aligned}
\|S p-p\| & \leq\left\|S p-S u^{k}\right\|+\left\|S u^{k}-u^{k}\right\|+\left\|u^{k}-p\right\| \\
& \leq\left\|p-u^{k}\right\|+\left\|S u^{k}-u^{k}\right\|+\left\|u^{k}-p\right\| \\
& =2\left\|u^{k}-p\right\|+\left\|S u^{k}-u^{k}\right\|,
\end{aligned}
$$

from (4.9) and (4.11) we get that $\|S p-p\|=0$, that is, $p \in \operatorname{Fix}(S)$.

From (3.16) we have

$$
\lim _{k \rightarrow \infty} \eta_{k}\left\|x^{k}-y^{k}\right\|^{2}=0
$$

We now consider two distinct cases.

Case 1 . $\limsup _{k \rightarrow \infty} \eta_{k}>0$.

Then there exist $\bar{\eta}>0$ and a subsequence $\left\{\eta_{k_{i}}\right\} \subset\left\{\eta_{k}\right\}$ such that $\eta_{k_{i}}>\bar{\eta}$ for all $i$. So we get from (4.12) that

$$
\lim _{i \rightarrow \infty}\left\|x^{k_{i}}-y^{k_{i}}\right\|=0
$$

Since $x^{k} \rightarrow p$, (4.13) implies that $y^{k_{i}} \rightarrow p$ as $i \rightarrow \infty$.

For each $y \in C$, we get from (3.20) that

$$
f\left(x^{k_{i}}, y\right)-f\left(x^{k_{i}}, y^{k_{i}}\right)+\frac{1}{\rho_{k_{i}}}\left\|y^{k_{i}}-x^{k_{i}}\right\|\left\|y-y^{k_{i}}\right\| \geq 0 .
$$

Letting $i \rightarrow \infty$, by the continuity of $f$, since $x^{k_{i}} \rightarrow p$ and $y^{k_{i}} \rightarrow p$, in the limit, from (4.14) we obtain that

$$
f(p, y)-f(p, p) \geq 0
$$

Hence,

$$
f(p, y) \geq 0, \quad \forall y \in C,
$$

so $p$ is a solution of $\operatorname{EP}(C, f)$. 
Case $2 . \lim _{k \rightarrow \infty} \eta_{k}=0$.

From the boundedness of $\left\{y^{k}\right\}$ we deduce that there exists $\left\{y^{k_{i}}\right\} \subset\left\{y^{k}\right\}$ such that $y^{k_{i}} \rightarrow \bar{y}$ as $i \rightarrow \infty$.

Replacing $y$ by $y^{k_{i}}$ in (3.19), we get

$$
f\left(x^{k_{i}}, y^{k_{i}}\right)+\frac{1}{\rho_{k_{i}}}\left\|y^{k_{i}}-x^{k_{i}}\right\|^{2} \leq 0 .
$$

In the other hand, by the Armijo linesearch rule (4.1), for $m_{k_{i}}-1$, there exists $z^{k_{i}, m_{k_{i}}-1}$ such that

$$
f\left(z^{k_{i}, m_{k_{i}}-1}, x^{k_{i}}\right)-f\left(z^{k_{i}, m_{k_{i}}-1}, y^{k_{i}}\right)<\frac{\theta}{2 \rho_{k_{i}}}\left\|y^{k_{i}}-x^{k_{i}}\right\|^{2} .
$$

Combining this with (4.15), we get

$$
f\left(z^{k_{i}, m_{k_{i}}-1}, y^{k_{i}}\right)-f\left(z^{k_{i}, m_{k_{i}}-1}, x^{k_{i}}\right)>-\frac{\theta}{2 \rho_{k_{i}}}\left\|y^{k_{i}}-x^{k_{i}}\right\|^{2} \geq \frac{2}{\theta} f\left(x^{k_{i}}, y^{k_{i}}\right) .
$$

According to the algorithm, we have $z^{k_{i}, m_{k_{i}}-1}=\left(1-\eta^{m_{k_{i}}-1}\right) x^{k_{i}}+\eta^{m_{k_{i}}-1} y^{k_{i}}$. Since $\eta^{k_{i}, m_{k_{i}}-1} \rightarrow 0$, $x^{k_{i}}$ converges strongly to $p$, and $y^{k_{i}}$ converges weakly to $\bar{y}$, this implies that $z^{k_{i}, m_{k_{i}}-1} \rightarrow p$ as $i \rightarrow \infty$. Besides that, $\left\{\frac{1}{\rho_{k_{i}}}\left\|y^{k_{i}}-x^{k_{i}}\right\|^{2}\right\}$ is bounded, so, without loss of generality, we may assume that $\lim _{i \rightarrow+\infty} \frac{1}{\rho_{k_{i}}}\left\|y^{k_{i}}-x^{k_{i}}\right\|^{2}$ exists. Hence, we get in the limit (4.16) that

$$
f(p, \bar{y}) \geq-2 \lim _{i \rightarrow+\infty} \frac{1}{\rho_{k_{i}}}\left\|y^{k_{i}}-x^{k_{i}}\right\|^{2} \geq \theta f(p, \bar{y}) .
$$

Therefore, $f(p, \bar{y})=0$ and $\lim _{i \rightarrow+\infty}\left\|y^{k_{i}}-x^{k_{i}}\right\|^{2}=0$. By Case 1 it is immediate that $p \in$ $\operatorname{Sol}(C, f)$. So

$$
p \in \operatorname{Sol}(C, f) \cap \operatorname{Fix}(S) \text {. }
$$

We obtain from (4.11) that $\lim _{k \rightarrow+\infty} A v^{k}=A p$. Combining this with (4.9) yields

$$
\lim _{k \rightarrow+\infty} w^{k}=A p
$$

Moreover,

$$
\begin{aligned}
\|T A p-A p\| & \leq\left\|T A p-T w^{k}\right\|+\left\|T w^{k}-w^{k}\right\|+\left\|w^{k}-A p\right\| \\
& \leq\left\|A p-w^{k}\right\|+\left\|T w^{k}-w^{k}\right\|+\left\|w^{k}-A p\right\| \\
& =2\left\|w^{k}-A p\right\|+\left\|T w^{k}-w^{k}\right\| .
\end{aligned}
$$

In view of (4.10) and (4.18), we obtain $\|T A p-A p\|=0$. Hence, $A p \in \operatorname{Fix}(T)$.

In addition,

$$
\begin{aligned}
\left\|T_{\beta}^{g} A p-A p\right\| & \leq\left\|T_{\beta}^{g} A p-T_{\alpha_{k}}^{g} A v^{k}\right\|+\left\|T_{\alpha_{k}}^{g} A v^{k}-A v^{k}\right\|+\left\|A v^{k}-A p\right\| \\
& =\left\|T_{\beta}^{g} A p-T_{\alpha_{k}}^{g} A v^{k}\right\|+\left\|w^{k}-A v^{k}\right\|+\left\|A v^{k}-A p\right\|
\end{aligned}
$$




$$
\begin{aligned}
& \leq\left\|A v^{k}-A p\right\|+\frac{\left|\alpha_{k}-\beta\right|}{\alpha_{k}}\left\|T_{\alpha_{k}}^{g} A v^{k}-A v^{k}\right\|+\left\|w^{k}-A v^{k}\right\|+\left\|A v^{k}-A p\right\| \\
& =2\left\|A v^{k}-A p\right\|+\frac{\left|\alpha_{k}-\beta\right|}{\alpha_{k}}\left\|w^{k}-A v^{k}\right\|+\left\|w^{k}-A v^{k}\right\|,
\end{aligned}
$$

where the last inequality comes from Lemma 8 . Letting $k \rightarrow \infty$ and recalling that $\lim _{k \rightarrow+\infty} A v^{k}=A p$, from (4.9) we get

$$
\left\|T_{\alpha}^{g} A p-A p\right\|=0 .
$$

Therefore, $A p \in \operatorname{Fix}\left(T_{\alpha}^{g}\right)=\operatorname{Sol}(Q, g)$.

Hence,

$$
A p \in \operatorname{Sol}(Q, g) \cap \operatorname{Fix}(T) .
$$

Combining this with (4.17), we conclude that $p \in \Omega$. The proof is completed.

When $S=I_{\mathbb{H}_{1}}$ and $T=I_{\mathbb{H}_{2}}$, Algorithm 3 becomes as follows.

\section{Algorithm 4}

Initialization. Pick $x^{g} \in C_{0}=C$ and choose the parameters $\eta, \theta \in(0,1), 0<\underline{\rho} \leq \bar{\rho}$,

$\left\{\rho_{k}\right\} \subset[\underline{\rho}, \bar{\rho}], 0<\underline{\gamma} \leq \bar{\gamma}<2,\left\{\gamma_{k}\right\} \subset[\underline{\gamma}, \bar{\gamma}], 0<\alpha,\left\{\alpha_{k}\right\} \subset[\alpha,+\infty), \mu \in\left(0, \frac{1}{\|A\|}\right)$.

Iteration $k(k=0,1,2, \ldots)$. Having $x^{\bar{k}}$, do the following steps:

Step 1 . Solve the strongly convex program

$$
C P\left(x^{k}\right) \quad \min \left\{f\left(x^{k}, y\right)+\frac{1}{2 \rho_{k}}\left\|y-x^{k}\right\|^{2}: y \in C\right\}
$$

to obtain its unique solution $y^{k}$.

If $y^{k}=x^{k}$, then set $u^{k}=x^{k}$ and go to Step 4. Otherwise, go to Step 2 .

Step 2. (Armijo linesearch rule) Find $m_{k}$ as the smallest positive integer number $m$ such that

$$
\left\{\begin{array}{l}
z^{k, m}=\left(1-\eta^{m}\right) x^{k}+\eta^{m} y^{m} \\
f\left(z^{k, m}, x^{k}\right)-f\left(z^{k, m}, y^{k}\right) \geq \frac{\theta}{2 \rho_{k}}\left\|x^{k}-y^{k}\right\|^{2} .
\end{array}\right.
$$

Set $\eta_{k}=\eta^{m_{k}}, z^{k}=z^{k, m_{k}}$.

Step 3. Select $\xi^{k} \in \partial_{2} f\left(z^{k}, x^{k}\right)$ and compute $\sigma_{k}=\frac{f\left(z^{k}, x^{k}\right)}{\left\|\xi^{k}\right\|^{2}}, u^{k}=P_{C}\left(x^{k}-\gamma_{k} \sigma_{k} \xi^{k}\right)$.

Step 4. $w^{k}=T_{\beta_{k}}^{g} A u^{k}$.

Step 5. $t^{k}=P_{C}\left(u^{k}+\mu A^{*}\left(w^{k}-A u^{k}\right)\right)$.

Step 6. Define $C_{k+1}=\left\{x \in C_{k}:\left\|x-t^{k}\right\| \leq\left\|x-u^{k}\right\| \leq\left\|x-x^{k}\right\|\right\}$. Compute $x^{k+1}=P_{C_{k+1}}\left(x^{g}\right)$ and go to iteration $k$ with $k$ is replaced by $k+1$.

The following result is an immediate consequence of Theorem 2.

Corollary 2 Let $g: Q \times Q \rightarrow \mathbb{R}$ be a bifunction satisfying Assumption $\mathrm{A}$, and $: C \times C \rightarrow \mathbb{R}$ be a bifunction satisfying Assumption $\mathrm{B}$. Let $A: \mathbb{H}_{1} \rightarrow \mathbb{H}_{2}$ be a bounded linear operator with its adjoint $A^{*}$. If $\Omega=\left\{x^{*} \in \operatorname{Sol}(C, f): A x^{*} \in \operatorname{Sol}(Q, g)\right\} \neq \emptyset$, then the sequences $\left\{x^{k}\right\}$ and $\left\{u^{k}\right\}$ converge strongly to an element $p \in \Omega$, and $\left\{w^{k}\right\}$ converges strongly to $A p \in \operatorname{Sol}(Q, g)$. 


\section{Conclusion}

Two linesearch algorithms for solving a split equilibrium problem and nonexpansive mapping $\operatorname{SEPNM}(C, Q, A, f, g, S, T)$ in Hilbert spaces have been proposed, in which the bifunction $f$ is pseudomonotone on $C$ with respect to its solution set, the bifunction $g$ is monotone on $Q$, and $S$ and $T$ are nonexpansive mappings. The weak and strong convergence of iteration sequences generated by the algorithms to a solution of this problem are obtained.

Competing interests

The authors declare that they have no competing interests.

\section{Authors' contributions}

All authors contributed equally and significantly in writing this article. All authors read and approved the final manuscript.

\section{Author details}

${ }^{1}$ Faculty of Information Technology, Le Quy Don Technical University, Hanoi, Vietnam. ${ }^{2}$ Department of Applied Mathematics, Pukyong National University, Busan, Korea. ${ }^{3}$ Technical University, Hanoi, Vietnam.

\section{Acknowledgements}

This work was supported by a Research Grant of Pukyong National University (2016).

Received: 12 November 2015 Accepted: 29 February 2016 Published online: 09 March 2016

\section{References}

1. Censor, Y, Elfving, T: A multiprojection algorithm using Bregman projections in a product space. Numer. Algorithms 8 , 221-239 (1994)

2. Byrne, C: A unified treatment of some iterative algorithms in signal processing and image reconstruction. Inverse Probl. 18, 103-120 (2004)

3. Censor, Y, Bortfeld, T, Martin, B, Trofimov, A: A unified approach for inversion problem in intensity-modulated radiation therapy. Phys. Med. Biol. 51, 2353-2365 (2006)

4. Censor, Y, Elfving, T, Kopf, N, Bortfeld, T: The multiple-sets split feasibility problem and its applications for inverse problems. Inverse Probl. 21, 2071-2084 (2005)

5. Censor, Y, Motova, XA, Segal, A: Perturbed projections and subgradient projections for the multiple-sets split feasibility problem. J. Math. Anal. Appl. 327, 1244-1256 (2007)

6. Byrne, C: Iterative oblique projection onto convex sets and the split feasibility problem. Inverse Probl. 18, 441-453 (2002)

7. Xu, HK: Iterative methods for the split feasibility problem in infinite dimensional Hilbert spaces. Inverse Probl. 26, 105018 (2010)

8. Ansari, QH, Rehan, A: An iterative method for split hierarchical monotone variational inclusions. Fixed Point Theory Appl. 2015, $121(2015)$

9. Ansari, AH, Rehan, A, Wen, CF: Split hierarchical variational inequality problems and fixed point problems for nonexpansive mappings. Fixed Point Theory Appl. 2015, 274 (2015)

10. Censor, Y, Segal, A: The split common fixed point problem for directed operators. J. Convex Anal. 16, 587-600 (2009)

11. Cui, H, Wang, F: Iterative methods for the split common fixed point problem in Hilbert spaces. Fixed Point Theory Appl. 2014, 78 (2014)

12. Eslamian, M: General algorithms for split common fixed point problem of demicontractive mappings. Optimization (2015). doi:10.1080/02331934.2015.1053883

13. Kraikaew, R, Saejung, S: On split common fixed point problems. J. Math. Anal. Appl. 415, 513-524 (2014)

14. Moudafi, A: The split common fixed point problem for demicontractive mappings. Inverse Probl. 26, 055007 (2010)

15. Sitthithakerngkiet, K, Deepho, J, Kumam, P: A hybrid viscosity algorithm via modify the hybrid steepest descent method for solving the split variational inclusion and fixed point problems. Appl. Math. Comput. 250, 986-1001 (2015)

16. Moudafi, A: Split monotone variational inclusions. J. Optim. Theory Appl. 150, 275-283 (2011)

17. Censor, Y, Gibali, A, Reich, S: Algorithms for the split variational inequality problem. Numer. Algorithms 59, $301-323$ (2012)

18. Deepho, J, Kumm, W, Kumm, P: A new hybrid projection algorithm for solving the split generalized equilibrium problems and the system of variational inequality problems. J. Math. Model. Algorithms 13, 405-423 (2014)

19. Deepho, J, Martínez-Moreno, J, Kumam, P: A viscosity of Cesàro mean approximation method for split generalized equilibrium, variational inequality and fixed point problems. J. Nonlinear Sci. Appl. 9, 1475-1496 (2016)

20. Deepho, J, Martínez-Moreno, J, Sitthithakerngkiet, K, Kumam, P: Convergence analysis of hybrid projection with Cesàro mean method for the split equilibrium and general system of finite variational inequalities. J. Comput. Appl. Math. (2015). doi:10.1016/j.cam.2015.10.006

21. Blum, E, Oettli, W: From optimization and variational inequalities to equilibrium problems. Math. Stud. 63, 127-149 (1994)

22. Muu, LD, Oettli, W: Convergence of an adaptive penalty scheme for finding constrained equilibria. Nonlinear Anal. TMA 18, 1159-1166 (1992)

23. He, Z: The split equilibrium problem and its convergence algorithms. J. Inequal. Appl. (2012). doi:10.1186/1029-242X-2012-162 
24. Bnouhachem, A, Al-Homidan, S, Ansari, QH: An iterative method for common solutions of equilibrium problems and hierarchical fixed point problems. Fixed Point Theory Appl. 2014, 194 (2014)

25. Kumam, W, Piri, H, Kumam, P: Solutions of system of equilibrium and variational inequality problems on fixed points of infinite family of nonexpansive mappings. Appl. Math. Comput. 248, 441-455 (2014)

26. Kumam, W, Witthayarat, U, Kumam, P, Suantai, S, Wattanawitoon, K: Convergence theorem for equilibrium problem and Bregman strongly nonexpansive mappings in Banach spaces. Optimization 65, 265-280 (2016)

27. Bauschke, HH, Borwein, JM: On projection algorithms for solving convex feasibility problems. SIAM Rev. 38, 367-426 (1996)

28. López, G, Martín-Márquez, V, Wang, F, Xu, HK: Solving the split feasibility problem without prior knowledge of matrix norms. Inverse Probl. 28, 085004 (2012)

29. Tran, DQ, Muu, LD, Nguyen, VH: Extragradient algorithms extended to equilibrium problems. Optimization 57 749-776 (2008)

30. Dinh, BV, Muu, LD: A projection algorithm for solving pseudomonotone equilibrium problems and it's application to a class of bilevel equilibria. Optimization 64, 559-575 (2015)

31. Facchinei, F, Pang, JS: Finite-Dimensional Variational Inequalities and Complementarity Problems. Springer, New York (2003)

32. Korpelevich, GM: An extragradient method for finding saddle points and other problems. Ėkon. Mat. Metody 12 747-756 (1976)

33. Mann, WR: Mean value methods in iteration. Proc. Am. Math. Soc. 4, 506-610 (1953)

34. Anh, TV, Muu, LD: A projection-fixed point method for a class of bilevel variational inequalities with split fixed point constraints. Optimization (2015). doi:10.1080/02331934.2015.1101599

35. Moudafi, A: Krasnoselski-Mann iteration for hierarchical fixed-point problems. Inverse Probl. 23, 1635-1640 (2007)

36. Takahashi, W, Takeuchi, Y, Kubota, R: Strong convergence theorems by hybrid methods for families of nonexpansive mappings in Hilbert spaces. J. Math. Anal. Appl. 341, 276-286 (2008)

37. Censor, Y, Gibali, A, Reich, S: Strong convergence of subgradient extragradient methods for variational inequality problem in Hilbert space. Optim. Methods Softw. 26, 827-845 (2011)

38. Opial, Z: Weak convergence of the sequence of successive approximations for nonexpansive mappings. Bull. Am. Math. Soc. 73, 591-597 (1967)

39. Rockafellar, RT: Convex Analysis. Princeton University Press, Princeton (1970)

40. Vuong, PT, Strodiot, JJ, Nguyen, VH: Extragradient methods and linesearch algorithms for solving Ky Fan inequalities and fixed point problems. J. Optim. Theory Appl. 155, 605-627 (2013)

41. Combettes, PL, Hirstoaga, A: Equilibrium programming in Hilbert spaces. J. Nonlinear Convex Anal. 6, 117-136 (2005)

\section{Submit your manuscript to a SpringerOpen ${ }^{\circ}$ journal and benefit from:}

- Convenient online submission

Rigorous peer review

- Immediate publication on acceptance

- Open access: articles freely available online

- High visibility within the field

- Retaining the copyright to your article 